\title{
Chronic pain after caesarean section: prevalence, impact and risk factors
}

Oliveira S.* , Portugal F.* , Lanzaro C.* , Pratas M.* , Anastácio M.* , Gomes P.*

*Centro Hospitalar e Universitário de Coimbra

\section{Background and Goals}

Chronic post-surgical pain (CPSP), as defined by International Association for the Study of Pain, is pain that persists for morte than 3 months after surgery and other causes are excluded. The caesarean section (CS) is one of the most performed surgeries currently and it is therefore fundamental to know the incidence and impact of chronic pain after CS and how to prevent it The aim of this study was to examine the prevalence and impact of CPSP after CS and to evaluate potential risk factors.

\section{MATERIALS AND METHODS}

-Women undergoing CS between January and June 2016 were included and interviewed by telephone one year after and data from our Acute Pain Unit database was consulted.

-Questions were about preoperative demographic and psychological factors, intraoperative clinical factors, acute postoperative pain and pain after 3, 6 and 12 months.

-Pain was evaluated with Visual Analogical Scale (VAS) from 0 to 10 and we used Brief Pain Inventory (BPI) to assess impact on daily life. We considered CPSP if VAS $>0$ after 3 months.

\section{RESULTS AND DISCUSSION}

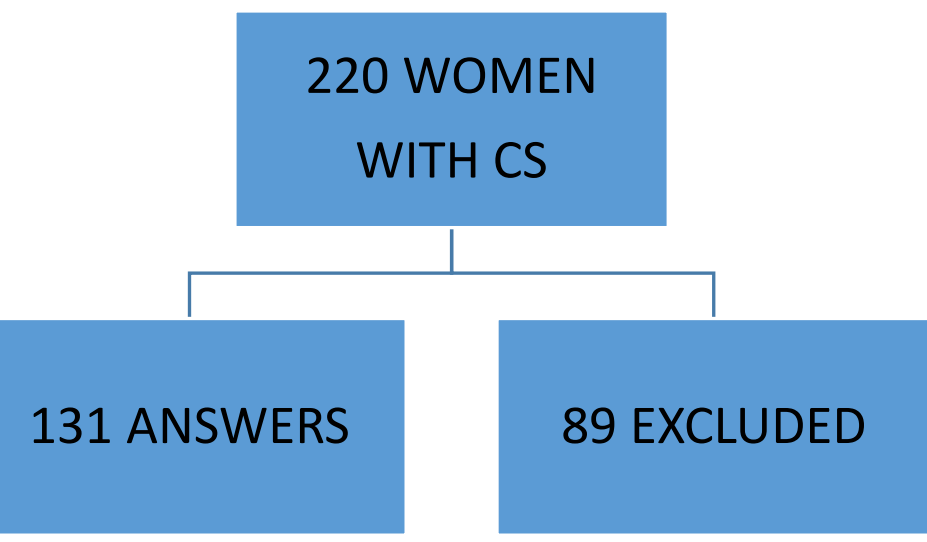

Table 1 - Sample physical description

\begin{tabular}{l|l}
\multicolumn{1}{c|}{ Variable } & \multicolumn{1}{c}{$\begin{array}{c}\text { Median (minimum - maximum) or } \\
\text { Mean } \pm \text { Standard deviation (SD) }\end{array}$} \\
\hline Age & $33,0 \pm 3,7$ years \\
\hline Gestacional age & $38(30-42)$ weeks \\
\hline Weight at end of pregnancy & $77,5 \pm 12,3 \mathrm{~kg}$ \\
\hline Height & $1,64 \pm 0,1 \mathrm{~m}$ \\
\hline Body mass index & $28,8 \pm 4,4 \mathrm{~kg} / \mathrm{m}^{2}$
\end{tabular}

Table 2 - Preoperative demographic and psychological factors, intraoperative clinical factors, post-operative clinical factors

\begin{tabular}{l|l}
\hline \multicolumn{1}{|c|}{ Variable } & \multicolumn{1}{c}{$\mathbf{N}(\%)$} \\
\hline Education & $\begin{array}{l}\text { High School: } 32(24,4 \%) \\
\text { Bachelor: } 52(39,7 \%\end{array}$ \\
\hline Depression / anxiety & $\begin{array}{l}\text { Depression : } 9(6,9 \%) / \text { Anxiety: } 7 \\
(5,3 \%)\end{array}$ \\
\hline Previous abdominal surgery / CS & $26(19,8 \%)$ \\
\hline Previous chronic pain & $15(11,5 \%)$ \\
\hline Induced labour & $32(24,4 \%)$ \\
\hline Closing technique & Staple : 84 (64,1\%) \\
\hline Elective/ emergent & Intradermic: $36(27,5 \%)$ \\
\hline Labour analgesia & Elective: $32(24,4 \%)$ \\
\hline Breastfeeding & Emergent : $99(75,6 \%)$ \\
\hline Post-partum depression & $70(53,4 \%)$ \\
\hline Paresthesia & $109(83,2 \%)$ \\
\hline Infection & $9(6,9 \%)$ \\
\hline & $18(13,7 \%)$ \\
\hline $5(3,8 \%)$
\end{tabular}

Table 3 - Pain assessment during hospitalization

\begin{tabular}{l|l|}
\hline Variable & Mean \pm SD \\
\hline VAS at rest within the first 24 hours & $5,4 \pm 2,1$ \\
\hline VAS on movement on the first 24 hours & $6,4 \pm 2,2$ \\
\hline VAS during stay & $4,1 \pm 2,0$
\end{tabular}

Graphic 1- Analgesic protocol distribution

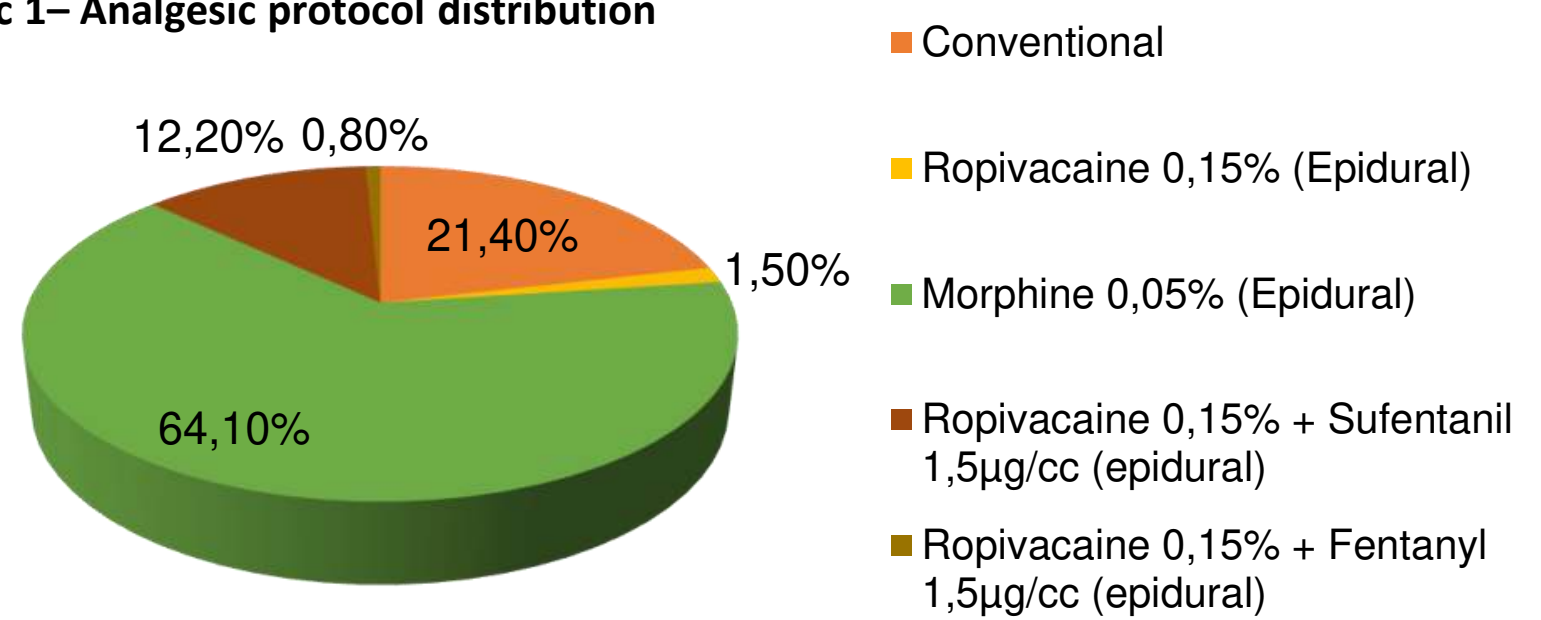

Graphic 2-Analgesic/ Anesthetic technique distribution

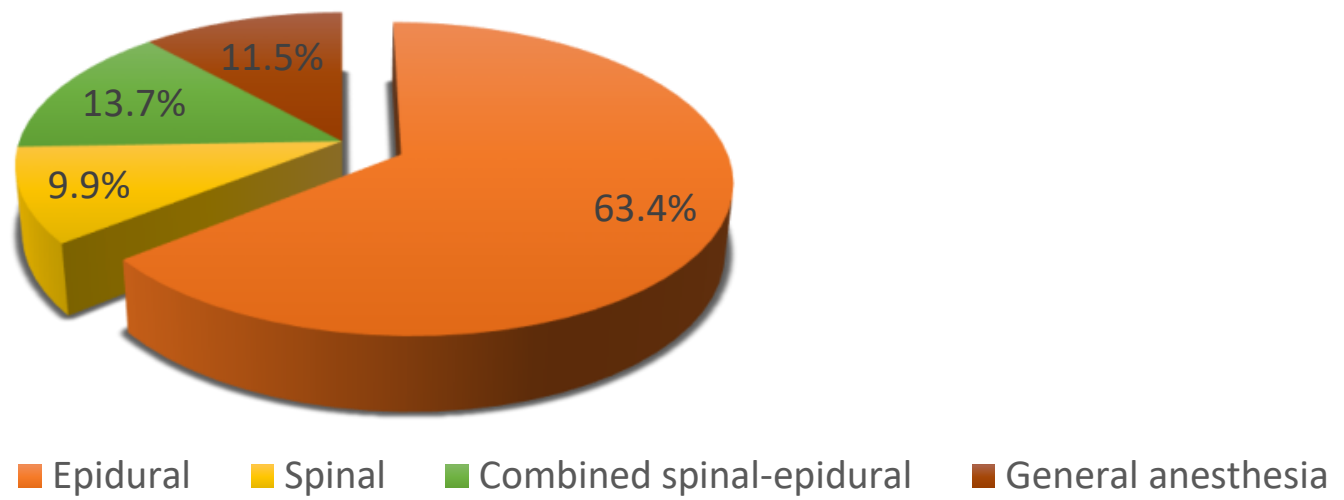

Table 4 - Pain assessment after discharge

\begin{tabular}{|l|l|l|}
\hline Variable & $\mathrm{N}(\%)$ & $\begin{array}{l}\text { Median (minimum - } \\
\text { maximum) }\end{array}$ \\
\hline VAS 3rd month & $\mathbf{2 5 ( 1 9 , 1 \% )}$ & $\mathbf{3 , 0}(\mathbf{2}-\mathbf{8})$ \\
\hline VAS 6th month & $19(14,5 \%)$ & $3,0(1-8)$ \\
\hline Current VAS & $11(8,4 \%)$ & $2,0(1-6)$
\end{tabular}

Table 5 - Comparison of chronic pain with different variables

\begin{tabular}{l|l|}
\hline Variable & P value \\
\hline Gestacional age & $\mathbf{P}=\mathbf{0 . 0 3 0}$ \\
\hline VAS on movement on the first 24 hours & $\mathbf{P}=\mathbf{0 . 0 4 4}$
\end{tabular}

\section{CONCLUSION}

- Although prevalence of CPSP after CS was 19\%, for most women included it did not have impact in their lives.

-Women with higher average pain intensity on movement within $24 \mathrm{~h}$ postoperatively have a greater risk of CPSP after CS and therefore attempts to better manage postoperative pain are needed in CPSP prevention. 